\title{
FULLY DEVELOPED BUBBLY TWO-PHASE FLOW THROUGH A PIPE: AN ANALYTICAL SOLUTION
}

\author{
WAQAS ALI ${ }^{1}$, MUBBASHAR NAZEER $^{2} \&$ AHMED ZEESHAN ${ }^{3}$ \\ ${ }^{1}$ Faculty of Engineering Technology, University of Twente, The Netherlands \\ ${ }^{2}$ Department of Mathematics, Riphah International University, Pakistan \\ ${ }^{3}$ Department of Mathematics and Statistics, International Islamic University Islamabad, Pakistan
}

\begin{abstract}
In this work, a novel homotopy analysis method for the solution of two-fluid models is presented. A fully developed bubbly through a pipe is considered. Different physical and mathematical properties of the two-fluid model were identified. The problem was solved in the central region of the pipe where the wall forces were neglected, which implies that void fractions and velocity profiles are not affected by the wall. The system of equations was reduced to a single equation without parameters with an intrinsic length scale L. Away from the wall of the pipe, the flat void fraction depends on the applied pressure gradient, the density of different phases and gravity. It was also found that the effective specific weight of the fluid column was cancelled by the pressure gradient.

Keywords: two-phase flow, laminar flow, bubbly flow, two-fluid model, fully developed flow.
\end{abstract}

\section{INTRODUCTION}

For the simulation of two-phase flows, the two-fluid model proposed by Ishii [1] is widely used. This model is based on the time averaging of two-phase field equations. The averaging process used in Ishii model needs to be accompanied with several closure approximations, which lack practical understanding. For example, lift term that models transversal force influencing particles or bubbles in a velocity field is not completely understood. In the current work, we analyse the two-fluid model by considering a fully developed bubbly flow in a circular pipe. Our main objective is to look into the generic properties of the model.

We first recall the two-fluid model in brief, with algebraic manipulations of the equations. The solution of the system is first provided away from the wall which follows the solution of the complete system.

\section{MATHEMATICAL FORMULATION}

We consider the mass and momentum conservation equations with no temperature effects similar to Drew and Passman [2], assumption.

$$
\begin{gathered}
\frac{\partial\left(\epsilon_{k} \rho_{k}\right)}{\partial t}+\nabla \cdot\left(\epsilon_{k} \rho_{k} \overrightarrow{v_{k}}\right)=0, \\
\frac{\partial\left(\epsilon_{k} \rho_{k} \overrightarrow{v_{k}}\right)}{\partial t}+\nabla \cdot\left(\epsilon_{k} \rho_{k} \overrightarrow{v_{k}} \overrightarrow{v_{k}}\right)=\nabla \cdot\left[\epsilon_{k}\left(T_{k}+\tau_{k}^{R e}\right)\right]+\epsilon_{k} \rho_{k} \vec{g}+M_{k},
\end{gathered}
$$

in which $\mathrm{k}$ is a phase ( $\mathrm{L}$ for liquid, $\mathrm{G}$ for gas), $\epsilon_{k}$ is the volumetric fraction of phase $\mathrm{k}, \overrightarrow{v_{k}}$ is the corresponding velocity, $\rho_{k}$ is the density, $T_{k}$ is the stress tensor, defined as

$$
T_{k}=-p_{k} I+\mu_{k}\left[\nabla \overrightarrow{v_{k}}+\left(\nabla \overrightarrow{v_{k}}\right)^{T}\right]
$$

where $p_{k}$ is the pressure, and $\mu_{k}$ is the viscosity $\tau_{k}^{R e}$ is the Reynolds like stress due to statistical fluctuations, modelled by Nigmatulin [3] 


$$
\tau_{k}^{R e}=-\epsilon_{G} \rho_{L}\left[A\left|\overrightarrow{v_{G}}-\overrightarrow{v_{L}}\right|^{2} I+B\left(\overrightarrow{v_{G}}-\overrightarrow{v_{L}}\right)\left(\overrightarrow{v_{G}}-\overrightarrow{v_{L}}\right)\right]
$$

where values for $\mathrm{A}$ and $\mathrm{B}$ are $\frac{3}{20}$ and $\frac{1}{20}$, respectively. Assume $\overrightarrow{\mathrm{V}}_{G}-\overrightarrow{\mathrm{V}}_{L}=\overrightarrow{\mathrm{V}}_{r}$ and define $\mathrm{V}_{r}=\left|\overrightarrow{\mathrm{V}}_{r}\right|$. In eqn (2), $M_{k}$ represents the interfacial momentum exchange, which relates to the actual force between phases $\left(M_{k}^{\prime}\right)$ according to

$$
M_{k}=p_{k i} \nabla \epsilon_{k}-\tau_{k i} . \nabla \epsilon_{k}+M_{k}^{\prime},
$$

where values at the interphase are represented by subscript $i$. Different models exist for $M_{k}$, in particular for the liquid phase $(k=L)$ Antal et al. [4] used

$$
M_{L}=p_{L i} \nabla \epsilon_{L}-\mu_{L}\left(\nabla v_{L}+\nabla v_{L}^{T}\right)+\rho_{L}\left(A\left|\vec{v}_{r}\right|^{2} I+B\left(\vec{v}_{r} \otimes \vec{v}_{r}\right)\right)+M_{L}^{\prime},
$$

whereas on the other hand Drew and Passman [2], used

$$
M_{L}=p_{L i} \nabla \epsilon_{L}-\left[\rho_{L}\left(a b\left|\vec{v}_{r}\right|^{2} I+a^{2}\left(\vec{v}_{r} \cdot \vec{v}_{r}\right)\right)\right] \nabla \epsilon_{L}-\rho_{L} \epsilon_{L} \nabla \cdot\left(\vec{v}_{r} \otimes \vec{v}_{r}\right)+M_{L}^{\prime},
$$

where $a=-\frac{9}{20}$ and $b=\frac{3}{20}$. The pressure at the interphase $\left(p_{L i}\right)$ can be derived from the expression by Stuhmiller [5],

$$
p_{L-} p_{L i}=C_{\rho_{L}} \epsilon_{L}\left|\vec{v}_{r}\right|^{2}, C=\frac{1}{4}
$$

We will consider drag, lift and wall forces,

$$
M_{L}^{\prime}=M_{K}^{\prime D}+M_{K}^{\prime L}+M_{K}^{\prime W},
$$

with

$$
\begin{aligned}
& M_{G}^{\prime D}=-M_{L}^{\prime D}=-\frac{3}{8} \frac{\epsilon_{G}}{R_{b}} C_{D \rho_{L}} \vec{v}_{r}\left|\vec{v}_{r}\right|, \\
& C_{D}=\frac{24}{R e}\left(1+0.1 R e^{0.75}\right) \text {, } \\
& R e=\frac{2 R_{b} \rho_{L}\left|\vec{v}_{r}\right|}{\mu_{m}}, \mu_{m}=\frac{\mu_{L}}{\left(1-\epsilon_{G}\right)^{\prime}} \\
& M_{L}^{L L}=-M_{L}^{\prime L}=-C_{L} \epsilon_{G} \rho_{L} \vec{v}_{r} \wedge\left(\nabla \wedge \vec{v}_{L}\right), \\
& M_{G}^{\prime W}=-M_{L}^{\prime W} \\
& =\left\{\begin{array}{rl}
\epsilon_{L} \rho_{L}\left|u_{\|}\right|^{2}\left[C_{w 1}+C_{w 2}\left(\frac{R_{b}}{y_{0}}\right)\right] \widetilde{n_{b}} \text { if } \frac{\epsilon_{G} \rho_{L}\left|u_{\|}\right|^{2}}{R_{b}}\left[C_{w 1}+C_{w 2}\left(\frac{R_{b}}{y_{0}}\right)\right]>0, \\
0 \quad \text { if } \frac{\epsilon_{G} \rho_{L}\left|u_{\|}\right|^{2}}{R_{b}}\left[C_{w 1}+C_{w 2}\left(\frac{R_{b}}{y_{0}}\right)\right] \leq 0
\end{array},\right. \\
& u_{\|}=\vec{v}_{r}-\left[\widetilde{n_{w}} \cdot \vec{v}_{r}\right] \text {, } \\
& C_{w 1}=-0.1, C_{w 2}=0.147 \text {, }
\end{aligned}
$$


where, $y_{0}$ is the distance to the wall, $\breve{n}_{w}$ the exterior unit normal and $R_{b}$ the bubble radius.

Considering incompressible unidirectional laminar fully developed flow of Newtonian fluid with bubble floating through a vertical cylindrical duct. The set of five differential equations corresponding to five unknown functions, i.e., $v_{L}(r), v_{G}(r), p_{L}(r, z), p_{G}(r, z)$ and $\epsilon_{G}(r, z)$, as follows.

Momentum conservation equation of the gas phase in the radial direction $\breve{r}$

$$
\epsilon_{G}\left[\frac{\partial p_{G}}{\partial r}+C_{L} \rho_{L} v_{r} \frac{\partial v_{L}}{\partial r}+\frac{\rho_{L} v_{r}^{2}}{R_{b}}\left(C_{w 1}+C_{w 2} \frac{R_{b}}{(R-r)}\right)\right]=0,
$$

where $R$ is the internal radius of the duct.

Momentum conservation equation of the gas phase in the axial direction $\breve{Z}$

$$
\frac{\partial p_{G}}{\partial r}=-\rho_{G} g-\frac{3}{8} \frac{C_{D}}{R_{b}} \rho_{L} v_{r}\left|v_{r}\right|
$$

Relative velocity $v_{r}$ is calculated by using the above equation, which is considered uniform. Momentum conservation equation of the liquid phase in the radial direction $\breve{r}$

$$
\begin{gathered}
\epsilon_{L} \frac{\partial p_{L}}{\partial r}=-\frac{\partial}{\partial r}\left[A \rho_{L} \epsilon_{L}\left(1-\epsilon_{L}\right) v_{r}^{2}\right]+C_{L} \epsilon_{G} \rho_{L} v_{r} \frac{\partial v_{L}}{\partial r}+C_{\rho_{L}}\left(1-\epsilon_{G}\right) v_{r}^{2} \frac{\partial \epsilon_{G}}{\partial r} \\
+\frac{\epsilon_{G} \rho_{L} v_{r}^{2}}{R_{b}}\left(C_{w 1}+C_{w 2} \frac{R_{b}}{(R-r)}\right)-C_{1} \rho_{L} v_{r}^{2} \frac{\partial \epsilon_{G}}{\partial r}
\end{gathered}
$$

Momentum conservation equation of the liquid phase in the axial direction $\breve{Z}$

$$
\epsilon_{L} \frac{\partial p_{L}}{\partial r}=\frac{1}{r} \frac{\partial}{\partial r}\left[r \epsilon_{L} \mu_{L} \frac{\partial v_{L}}{\partial r}\right]+\frac{3}{8} \frac{\epsilon_{G}}{R_{b}} C_{D} \rho_{L} v_{r}\left|v_{r}\right|-\epsilon_{L} \rho_{L} g+C_{2} \mu_{L} \frac{\partial v_{L}}{\partial r} \frac{\partial \epsilon_{G}}{\partial r} .
$$

Jump condition at the interphase is given by

$$
p_{G}-p_{L}=\frac{2 \sigma}{R_{b}}-C_{\rho_{L}}\left(1-\epsilon_{G}\right) v_{r}^{2}
$$

\section{PROBLEM SOLUTION}

3.1 Region away from the wall

We define

$$
\rho \equiv \rho_{e f f}=\epsilon_{L} \rho_{L}+\epsilon_{G} \rho_{G}, \bar{\rho} \equiv \frac{1}{s} \int \rho . d s, \quad \frac{\partial P}{\partial z} \equiv \frac{\partial p}{\partial z}+\bar{\rho} g,
$$

where $S$ is the cross-sectional area of the pipe.

Eliminating the drag force from eqns (18) and (20), and using $\epsilon_{L}=\frac{\left(\rho-\rho_{G}\right)}{\left(\rho_{L}-\rho_{G}\right)}$ we get

$$
\frac{\partial p}{\partial z}+(\rho-\bar{\rho}) g=\frac{\left(\rho-\rho_{G}\right)}{\left(\rho_{L}-\rho_{G}\right)} \frac{\mu_{L}}{r} \frac{\partial}{\partial r}\left(r \frac{\partial v_{L}}{\partial r}\right)+\left(C_{2}-1\right) \mu_{L} \frac{\partial v_{L}}{\partial r} \frac{\partial \epsilon_{G}}{\partial r} .
$$

On the other hand, replacing eqns (19) and (21) into eqn (17) we get, after multiplication by $\frac{\epsilon_{L}}{\left(\rho_{L} v_{r}\right)}$, 


$$
\begin{aligned}
\frac{\left(\rho-\rho_{G}\right)}{\left(\rho_{L}-\rho_{G}\right)} \frac{1}{\left(\rho_{L}-\rho_{G}\right)} \frac{\partial p}{\partial z}= & \frac{C_{L}}{(2 C-A) v_{r}} \frac{\partial v_{L}}{\partial r}+\frac{1}{(2 C-A) R_{b}}\left(C_{w 1}+C_{w 2} \frac{R_{b}}{(R-r)}\right) \\
& -\frac{\left(C_{1}+A \epsilon_{G}\right)}{(2 C-A)} \frac{1}{\left(\rho_{L}-\rho_{G}\right)} \frac{\partial p}{\partial r} .
\end{aligned}
$$

As a result, the system is reduced to only two equations. Introducing non-dimensional variables:

$$
\begin{gathered}
\frac{\partial p^{*}}{\partial z}=\frac{1}{\left(\rho_{L}-\rho_{G}\right)} \frac{\partial p}{\partial z}, L^{2}=\frac{2 \mu_{L} v_{L}(C-A)}{g\left(\rho_{L}-\rho_{G}\right) C_{L}}, v^{*}=\frac{v_{L}}{v_{r}} \frac{C_{L}}{2(C-A)}, r^{*}=\frac{r}{L}, R^{*}=\frac{R}{L}, R_{L}^{*}=\frac{R_{b}}{L}, E= \\
\frac{C_{w_{1}}}{2(C-A) R_{b}^{*}}, D=\frac{C_{w_{2}}}{2(C-A)} .
\end{gathered}
$$

From eqns (23) and (24), using the scaled variables in eqn (25) and assuming the wallforce terms are negligible, the system is reduced to following ordinary differential equation

$$
\begin{gathered}
\left(\epsilon_{L}\right)^{2} \frac{\partial^{2} \epsilon_{L}}{\partial r^{* 2}}+\left(2-C_{2}\right) \epsilon_{L}\left(\frac{\partial \epsilon_{L}}{\partial r^{*}}\right)^{2}+\frac{\left(\epsilon_{L}\right)^{2}}{r^{*}} \frac{\partial \epsilon_{L}}{\partial r^{*}}-\epsilon_{L}+\frac{\left(C_{1}+A\right)}{2(C-A)}\left[\epsilon_{L}\left(\frac{1}{r^{*}} \frac{\partial \epsilon_{L}}{\partial r^{*}}+\frac{\partial^{2} \epsilon_{L}}{\partial r^{* 2}}\right)-\right. \\
\left.\left(C_{2}-1\right)\left(\frac{\partial \epsilon_{L}}{\partial r^{*}}\right)^{2}\right]=\frac{\partial p^{*}}{\partial z}-\overline{\epsilon_{L}},
\end{gathered}
$$

where $\overline{\epsilon_{L}}=\frac{\left(\bar{\rho}-\rho_{G}\right)}{\left(\rho_{L}-\rho_{G}\right)}$. This equation is valid where the wall force is not active $\left(R-r>\frac{D L}{E}\right)$, It can be seen that the right-hand side is a constant, independent of $r^{*}$. The values of the constants $A$ and $B$ are fixed $\left(A=\frac{3}{20}, B=\frac{1}{20}\right)$, and considered following two set of values for $C_{1}$ and $C_{2}$, with $C_{1}=-\frac{3}{20}$ and $C_{2}=1$, taken for Antal et al. [4] (Model I), and $C_{1}=\frac{37}{100}$ and $C_{2}=0$, for Drew and Passman [2] (Model II).

In the case of Model I, eqn (26) is reduced to

$$
\left(\epsilon_{L}\right)^{2} \frac{\partial^{2} \epsilon_{L}}{\partial r^{* 2}}+\epsilon_{L}\left(\frac{\partial \epsilon_{L}}{\partial r^{*}}\right)^{2}+\frac{\left(\epsilon_{L}\right)^{2}}{r^{*}} \frac{\partial \epsilon_{L}}{\partial r^{*}}-\epsilon_{L}=\frac{\partial p^{*}}{\partial z}-\overline{\epsilon_{L}},
$$

which is the equation of laminar fully developed bubbly flow far from the wall and contains no parameter except constant on the right-hand side. Eqn (27) can be solved to get a different solution for pressure gradient and the amount of gas in the flow. By using $r^{*}=0, \varepsilon_{L}(0)$ eqn (27) can be rewritten as

$$
\frac{\partial P^{*}}{\partial z}-\overline{\epsilon_{L}}=-\epsilon_{L}(0)(1+\lambda)
$$

finally resulting in

$$
\left(\epsilon_{L}\right)^{2} \frac{\partial^{2} \epsilon_{L}}{\partial r^{* 2}}+\epsilon_{L}\left(\frac{\partial \epsilon_{L}}{\partial r^{*}}\right)^{2}+\frac{\left(\epsilon_{L}\right)^{2}}{r^{*}} \frac{\partial \epsilon_{L}}{\partial r^{*}}-\epsilon_{L}=-\epsilon_{L}(0)(1+\lambda)
$$

If the model of Drew and Passman is used we following equation is obtained

$$
\begin{aligned}
\left(\epsilon_{L}\right)^{2} \frac{\partial^{2} \epsilon_{L}}{\partial r^{* 2}}+2 \epsilon_{L}\left(\frac{\partial \epsilon_{L}}{\partial r^{*}}\right)^{2}+\frac{\left(\epsilon_{L}\right)^{2}}{r^{*}} \frac{\partial \epsilon_{L}}{\partial r^{*}}-\epsilon_{L} \\
+\frac{33}{80}\left[\epsilon_{L}\left(\frac{1}{r^{*}} \frac{\partial \epsilon_{L}}{\partial r^{*}}+\frac{\partial^{2} \epsilon_{L}}{\partial r^{*}}\right)+\left(\frac{\partial \epsilon_{L}}{\partial r^{*}}\right)^{2}\right]=-\epsilon_{L}(0)(1+\lambda),
\end{aligned}
$$


where $\lambda$ is a measure of the difference between the applied pressure gradient and the effective weight of the mixture at the centre of the pipe. With the given initial conditions $\epsilon_{L}(0)$ and $\epsilon_{L}^{\prime}(0)$ we then solve eqns (29) and (30).

\section{SOLUTION OF THE PROBLEM}

In the previous section problem in the region where the wall forces are not active. In addition, all applied pressure gradients were not considered as when $-\partial p_{L} / \partial \mathrm{z}$ is greater than $\rho_{L} \bar{g}$, at the centre by adjusting the void fraction, the pressure gradient cannot be balanced. This is valid even if the void fraction is assumed zero.

Eqns (17)-(21) can be solved for $v_{L}(r)$ and $\varepsilon_{G}(r)$, which after mathematical manipulation and elimination of drag coefficient results in

$$
\begin{gathered}
\frac{\partial p_{L}}{\partial z}+\left(1-\epsilon_{G}\right) \rho_{L} g+\epsilon_{G} \rho_{G} g=\frac{1}{r} \frac{\partial}{\partial r}\left[r\left(1-\epsilon_{G}\right) \mu_{L} \frac{\partial v_{L}}{\partial r}\right]+C_{2} \mu_{L} \frac{\partial v_{L}}{\partial r} \frac{\partial \epsilon_{G}}{\partial r}, \\
\epsilon_{G} \frac{\partial \epsilon_{G}}{\partial r} v_{r}^{2}\left[\left(\frac{1}{2}-A+C_{1}\right)-\frac{1}{5} \epsilon_{G}\right]=-\epsilon_{G} C_{L} v_{r} \frac{\partial v_{r}}{\partial r}-\epsilon_{G}\left(C_{w 1}+C_{w 2} \frac{R_{b}}{R r}\right) \frac{v_{r}^{2}}{R_{b}},
\end{gathered}
$$

with following boundary conditions

$$
\frac{\partial v_{L}}{\partial r}(0)=0, v_{L}(R)=0, \frac{\partial \epsilon_{G}}{\partial r}(0)=0 .
$$

Using the non-dimensional parameter defined in eqn (25) we get the following form

$$
\begin{gathered}
\frac{\partial P}{\partial z}+\frac{\rho_{L}}{\rho_{L}-\rho_{G}}-\epsilon_{G}=C_{2} \frac{\partial v}{\partial r} \frac{\partial \epsilon_{G}}{\partial r}+\frac{1}{r}\left(1-\epsilon_{G}\right) \frac{\partial v}{\partial r}+\frac{\partial v}{\partial r} \frac{\partial \epsilon_{G}}{\partial r}+\left(1-\epsilon_{G}\right) \frac{\partial^{2} v}{\partial r^{2}} \\
\epsilon_{G} \frac{\partial \epsilon_{G}}{\partial r} \frac{\left(\frac{1}{2}-A+C_{1}\right)}{2(C-A)}-\epsilon_{G}^{2} \frac{\partial \epsilon_{G}}{\partial r}\left(\frac{1}{10(C-A)}\right)=-\epsilon_{G} \frac{\partial v}{\partial r}-E \epsilon_{G}-\frac{D \epsilon_{G}}{R-r^{\prime}}
\end{gathered}
$$

subject to boundary conditions

$$
\begin{gathered}
\frac{\partial v_{L}}{\partial r}(0)=0, \quad v_{L}(R)=0, \quad \frac{\partial \epsilon_{G}}{\partial r}(0)=0, \\
\text { where } P=\frac{\partial P}{\partial z}+\frac{\rho_{L}}{\rho_{L}-\rho_{G}} .
\end{gathered}
$$

Eqns (34)-(36) are solved in Mathematica [6], for a wide set of values by using the homotopy analysis method (HAM). Zero-order deformation of the system of equations, the linear operator $L$ and the non-linear operator $N$ are defined as

$$
\begin{gathered}
L(f)=\frac{d^{2} f}{d r^{2}}, \\
L(g)=\frac{d g}{d r}, \\
N(f)=\frac{\partial p}{\partial z}+\frac{\rho_{L}}{\rho_{L}-\rho_{G}}-\epsilon_{G}-C_{2} \frac{\partial v}{\partial r} \frac{\partial \epsilon_{G}}{\partial r}-\frac{1}{r}\left(1-\epsilon_{G}\right) \frac{\partial v}{\partial r}-\frac{\partial v}{\partial r} \frac{\partial \epsilon_{G}}{\partial r} \\
-\left(1-\epsilon_{G}\right) \frac{\partial^{2} v}{\partial r^{2}},
\end{gathered}
$$


154 Computational and Experimental Methods in Multiphase and Complex Flow X

$$
N(g)=\epsilon_{G} \frac{\partial \epsilon_{G}}{\partial r} \frac{\left(\frac{1}{2}-A+C_{1}\right)}{2(C-A)}-\epsilon_{G}^{2} \frac{\partial \epsilon_{G}}{\partial r}\left(\frac{1}{10(C-A)}\right)+\epsilon_{G} \frac{\partial v}{\partial r}+E_{\epsilon_{G}}+\frac{D \epsilon_{G}}{R-r} .
$$

In the view of boundary condition, the initial guess and boundary condition are

$$
\begin{gathered}
f(0)=\frac{1}{4}\left(P r^{2}-P R^{2}\right), \\
g(0)=\frac{\left(r^{2}-R^{2}\right)}{2}, \\
f(R, h)=0 ; f(0, h)=0 ; g(0, h)=0, \\
L\left\{f_{m}(x)\right\}-\left(1-x_{m}\right) L\left\{f_{0}\right\}=X_{m} R_{1 m} \\
L\left\{g_{m}(x)\right\}-\left(1-x_{m}\right) L\left\{g_{0}\right\}=X_{m} R_{2 m},
\end{gathered}
$$

and the mth order deformation is

$$
\begin{aligned}
R_{1 m}\left(V_{m-1}, \epsilon_{m-1}\right)=\epsilon_{G m-1}(r)+C_{2} \sum_{j=0}^{m-1} V_{j}^{\prime}(r) \epsilon_{G m-1}^{\prime}(r)+\frac{1}{r} V_{m-1}^{\prime}(r) \\
-\sum_{j=0}^{m-1} \epsilon_{G j}(r) V_{m-1-j}^{\prime}(r)+\sum_{j=0}^{m-1} V_{j}^{\prime}(r) \epsilon_{G m-1-j}^{\prime}(r) \\
+V_{m-1}^{\prime \prime}+\sum_{j=0}^{m-1} \epsilon_{G j}(r) V_{m-1-j}^{\prime \prime}(r)-X_{m} \lambda^{\prime} \\
R_{2 m}\left(V_{m-1}, \epsilon_{m-1}\right)=\frac{\left(\frac{1}{2} A+C_{1}\right)}{2(C-)} \sum_{j=0}^{m-1} \epsilon_{G j}(r) \epsilon_{G m-1-j}^{\prime}(r) \\
-\frac{1}{10(C-A)} \sum_{k=0}^{m-1} \sum_{j=0}^{m-1} \epsilon_{G k}(r) \epsilon_{G j-k}(r) \epsilon_{G m-1-j}^{\prime}(r) \\
+\sum_{j=0}^{m-1} \epsilon_{G j}(r) V_{m-1-j}^{\prime}(r)+E_{\epsilon G m-1}(r)+\frac{D}{R-r} \epsilon_{G m-1}(r),
\end{aligned}
$$

where

$$
X_{m}=\left\{\begin{array}{c}
0 \quad \text { when } m \leq 1 \\
0 \quad \text { otherwise }
\end{array}\right.
$$

Zero-order and first-order solution by using HAM are

$$
\begin{gathered}
f_{0}=\frac{R^{4}}{12}-\frac{P R^{4}}{30}-\frac{R^{2 x^{2}}}{10}+\frac{x^{4}}{60}+\frac{P x^{4}}{30}+\frac{1}{4}\left(-P R^{2}+P x^{2}\right) \\
g_{0}=\frac{147 x^{2}}{2000}-\frac{R^{2 x^{2}}}{10}-\frac{P R^{2 x^{2}}}{20}-\frac{R^{4 x^{2}}}{20}+\frac{49 L x^{3}}{500 R_{b}}+\frac{3 x^{4}}{20}+\frac{3 P x^{4}}{40}-\frac{3 R^{2 x^{4}}}{20}-\frac{x^{6}}{12} \\
+\frac{1}{2}\left(-R^{2}+x^{2}\right)
\end{gathered}
$$




$$
\begin{aligned}
f_{1}=\frac{43241 R^{4}}{180000}- & \frac{7159 P R^{4}}{90000}+\frac{16 R^{6}}{1125}-\frac{7 P R^{6}}{4500}-\frac{P^{2} R^{6}}{1500}+\frac{11 R^{8}}{42000}-\frac{23 P R^{8}}{21000} \\
& -\frac{49 L R^{5}}{25000 R_{b}}-\frac{147 L P R^{5}}{25000 R_{b}}-\frac{7 R^{4} x^{2}}{25}+\frac{7159 x^{4}}{180000}+\frac{7159 P x^{4}}{90000} \\
& -\frac{17 R^{2} x^{4}}{900}-\frac{23 P R^{2} x^{4}}{1800}-\frac{P^{2} R^{4} x^{4}}{300}-\frac{R^{4} x^{4}}{600}+\frac{49 L x^{5}}{25000 R_{b}} \\
& +\frac{147 L P x^{5}}{25000 R_{b}}+\frac{7 x^{6}}{1500}+\frac{43 P x^{6}}{3000}+\frac{P^{2} x^{6}}{250}+\frac{R^{2} x^{6}}{600}+\frac{P R^{2} x^{6}}{300}-\frac{x^{8}}{1680} \\
& -\frac{P x^{8}}{280}+\frac{\left(-P R^{2}+P x^{2}\right)}{4}
\end{aligned}
$$

$$
\begin{aligned}
& g_{1}=-\frac{272391 x^{2}}{2000000}-\frac{3853 R^{2} x^{2}}{20000}-\frac{4147 P R^{2} x^{2}}{40000}-\frac{551 R^{4} x^{2}}{10000}+\frac{P R^{4} x^{2}}{1000}+\frac{R^{6} x^{2}}{50} \\
& +\frac{P R^{6} x^{2}}{200}+\frac{R^{8} x^{2}}{200}-\frac{7203 L R x^{2}}{500000 R_{b}}-\frac{147 R x^{3}}{5000}-\frac{147 P R x^{3}}{10000}-\frac{49 R^{3} x^{3}}{3750} \\
& +\frac{81193 L x^{3}}{500000 R_{b}}-\frac{147 L R^{2} x^{3}}{2500 R_{b}}-\frac{49 L P R^{2} x^{3}}{5000 R_{b}}-\frac{147 L P R^{4} x^{3}}{5000 R_{b}} \\
& +\frac{8913 x^{4}}{40000}+\frac{1959 P x^{4}}{16000}+\frac{32 R^{2} x^{4}}{625}-\frac{11 P R^{2} x^{4}}{100}-\frac{3 P^{2} R^{2} x^{4}}{400} \\
& -\frac{9 R^{4} x^{4}}{40}-\frac{3 P R^{4} x^{4}}{50}-\frac{3 R^{6} x^{4}}{40}+\frac{21609 L^{2} x^{4}}{1000000 R_{b}}+\frac{49 R x^{5}}{2500} \\
& +\frac{1421 L x^{5}}{12500 R_{b}}+\frac{833 L P x^{5}}{25000 R_{b}}+\frac{1421 L R^{2} x^{5}}{12500 R_{b}}+\frac{929 x^{6}}{18000}+\frac{11 P x^{6}}{90} \\
& +\frac{P^{2} x^{6}}{80}+\frac{13 R^{2} x^{6}}{30}+\frac{P R^{2} x^{6}}{8}+\frac{13 R^{4} x^{6}}{60}-\frac{399 L x^{7}}{5000 R_{b}}-\frac{133 x^{8}}{600} \\
& -\frac{161 P x^{8}}{2400}-\frac{161 P x^{8}}{2400}-\frac{133 R^{2} x^{8}}{600}+\frac{3 x^{10}}{40}+\frac{21609 R^{2} x}{1000000(R+x)} \\
& -\frac{147 R^{4} x}{10000(R+x)}-\frac{147 P R^{4} x}{20000(R+x)}-\frac{7203 L R^{3} x}{250000 R_{b}(R+x)} \\
& +\frac{\left(-R^{2}+x^{2}\right)}{2}-\frac{21609 R^{2} \log [-R+x]}{1000000}+\frac{147 R^{4} \log [-R+x]}{10000} \\
& +\frac{147 P R^{4} \log [-R+x]}{20000}+\frac{49 R^{6} \log [-R+x]}{10000} \\
& +\frac{7203 \operatorname{LR} R^{3} \log [-R+x]}{250000 R_{b}},
\end{aligned}
$$

and so on.

\section{RESULTS AND DISCUSSION}

Analysis of the two-fluid model in case of upward bubbly flows shows that there exists a socalled peak in the void fraction in the close vicinity of the wall. It is evident that, for the gasliquid mixture to flow upward, the pressure gradient must overcome the horizontally averaged weight $\bar{\rho} \bar{g}$. It is clear that the bubble should vanish on the wall. 
Figs 1 and 2 show upward flow which is away from the wall for the different values of $\lambda$. The greater value of $\lambda$ indicates, greater pressure gradient as compared to effective weight. Graphs for model I and model II are shown and upward motion is rapid for large values of $\lambda$. Fig. 3 shows a comparison of the model I and model II. It can be seen that both models converge approximately to the same value.

Figs 4-6, show the downward flow of void space for model I and model II for different values of $\epsilon_{G}(0)$. The gravity effect dominates pressure gradient and bubble moves towards the lower wall. Figs 7-9 shows the variation of initial coordinate $\epsilon_{G}(0)$, on both model I and model II. For upward flow a greater value of $\epsilon_{G}(0)$, show a quick rise of the bubble. Close to the wall, forces are effective, eqns (33)-(35) in non-dimensional form are simulated by using homotopy analysis method. The results shown in Figs 10-12 are for different values of bubble radius $R_{b}$, intrinsic length $L$ and $\lambda^{\prime}$. It is seen that void fraction profile decreases as the absolute value of applied pressure gradient increases, peak enlarges and radical position of maximum moves towards the wall. This is due to the result of the rise in lift force that comply to increasing liquid velocity gradient at the region close to the wall.

In fully developed laminar flow the solutions of the two-fluid model are such that the effective weight at the centre of the pipe (i.e., $\rho_{\text {eff }} \vec{g}$ ) practically balances the applied pressure gradient. Also at some particular limiting value of $r=r^{*}$ called critical radius at which solution is unreal, i.e., the value of liquid fraction either become zero or greater than one.

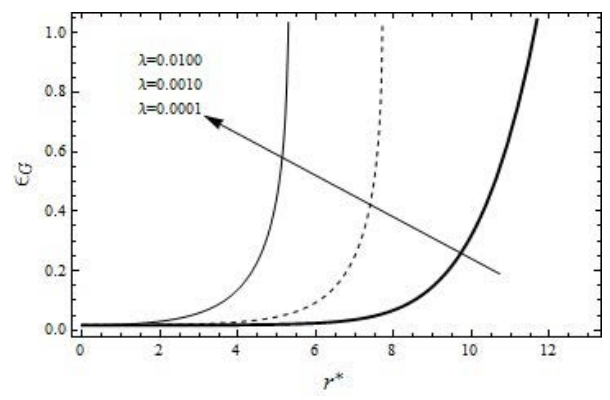

Figure 1: Upward flow for model I.

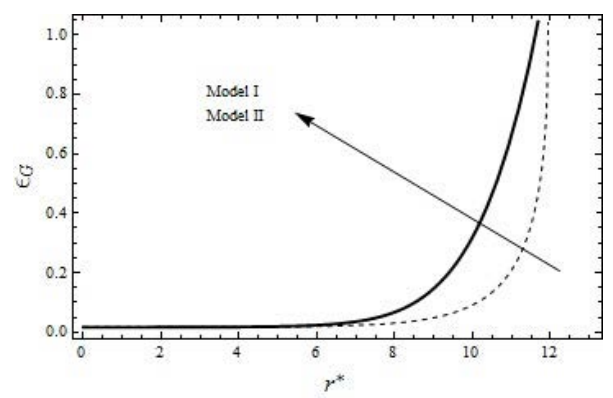

Figure 3: Upward flow for $\lambda=0: 0001$.

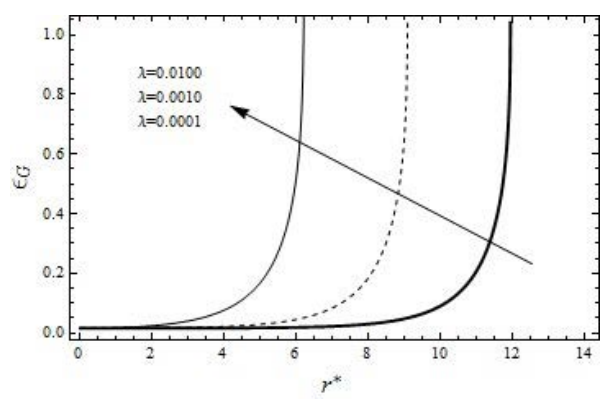

Figure 2: Upward flow for model II.

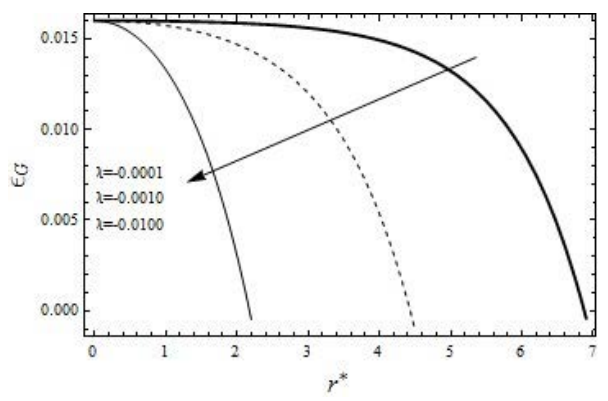

Figure 4: Downward flow for model I. 


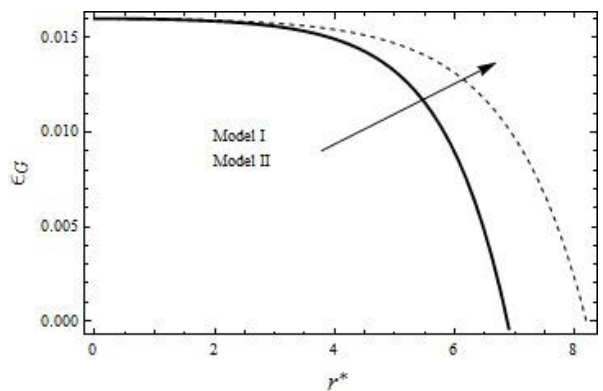

Figure 5: Downward flow for model II.

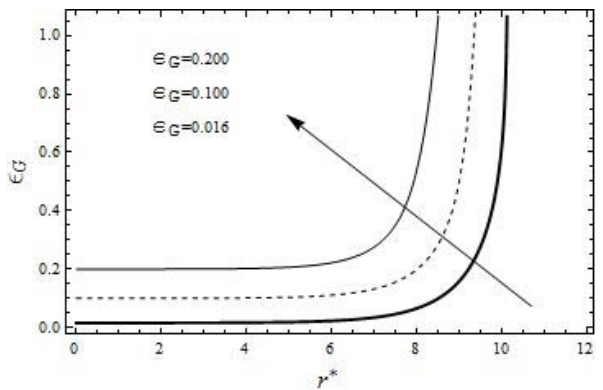

Figure 7: $\varepsilon_{G}\left(r^{*}\right)$ for different $\varepsilon_{G}(0)$ model I.

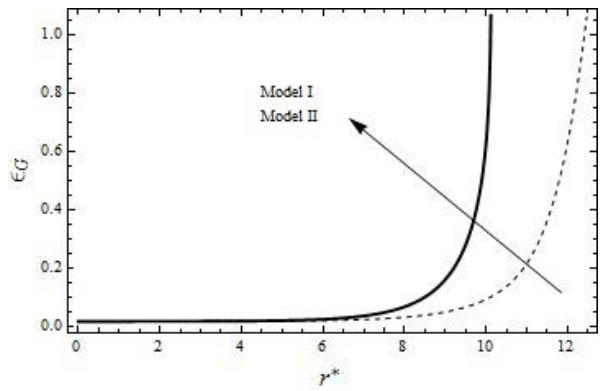

Figure 9: Comparison of $\varepsilon_{G}\left(r^{*}\right)$ for model I and model II for $\varepsilon_{G}(0)=0.016$.

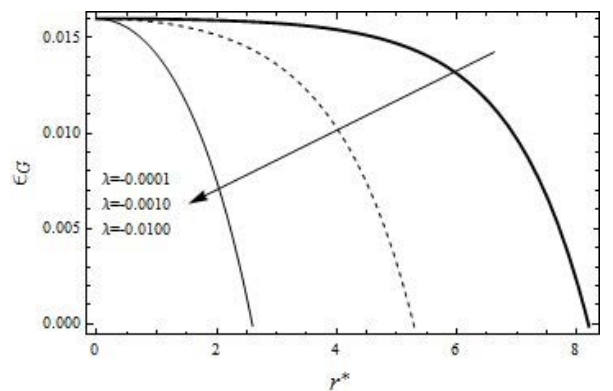

Figure 6: Comparison of downward flow for model I and model II for $\lambda=0.0001$.

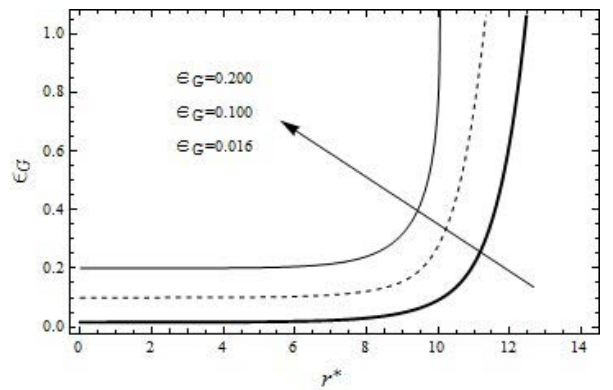

Figure 8: $\varepsilon_{G}\left(r^{*}\right)$ for different $\varepsilon_{G}(0)$ model II.

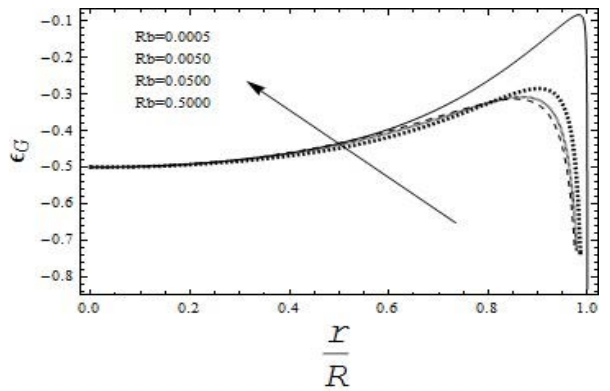

Figure 10: Void fraction profile variation in $R_{b}$. 

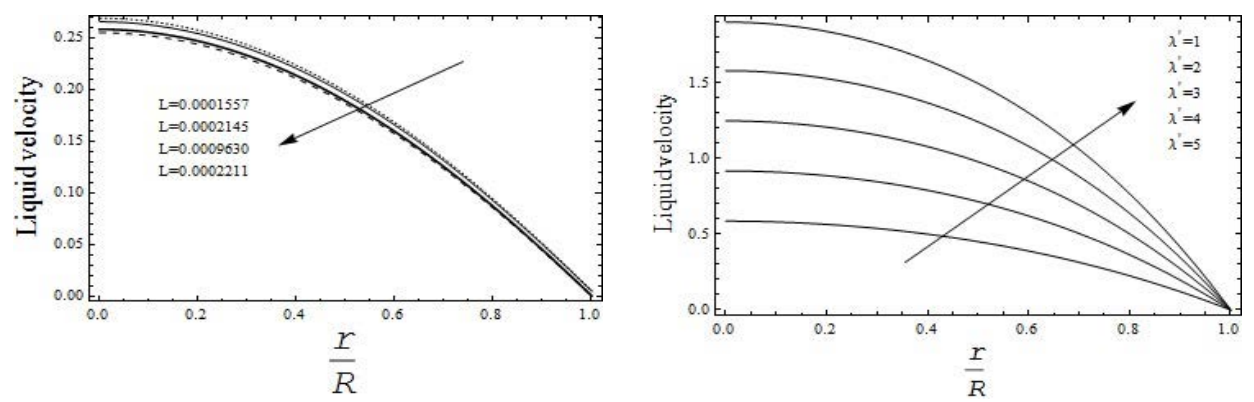

Figure 11: Velocity profile for variation of $L$. Figure 12: Liquid velocity profile for variation of upward flow.

In current work several physical and mathematical properties of the two-fluid model in the simple case of a laminar fully developed bubbly flow in case of a circular pipe have been identified. In vertical two-phase flow, it is found that the pressure gradient is cancelled with the effective specific weight of the fluid column. It is also found that the lift force balances the applied pressure gradient. For reduced gravity values, a prominent effect of lift force was observed. In future work, the effect of different boundary conditions on the model will be studied.

\section{REFERENCES}

[1] Ishii, M., Thermo-Fluid Dynamic Theory of Two-Phase Flow, Direction des Etudes et Recherches d'ElectricitXe de France: Eyrolles, 1975.

[2] Drew, D. \& Passman, S., Theory of Multicomponent Fluids, Springer: Berlin, 1998.

[3] Nigmatulin, R., Spatial averaging in the mechanics of heterogeneous and dispersed systems. International Journal of Multiphase Flow, 4, pp. 353-385, 1979.

[4] Antal, S., Lahey, R. Jr. \& Flaherty, J., Analysis of phase distribution in fully developed laminar bubbly two-phase flow. International Journal of Multiphase Flow, 17(5), pp. 635-652, 1991.

[5] Stuhmiller, J., The influence of interfacial pressure on the character of two-phase flow model equations. ASME Symposium on Computational Techniques for Non-Equilibrium Two-Phase Phenomena, p. 118, 1977.

[6] Wolfram, S., Mathematica, Addison-Wesley: Reading, MA, 1991. 